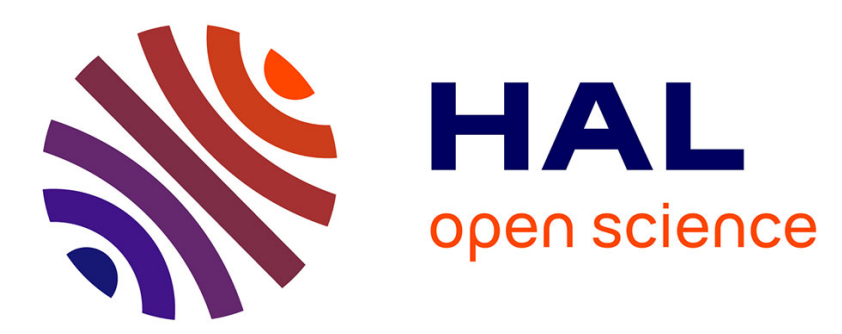

\title{
A fast lesion registration to assist coronary heart disease diagnosis in CTA images
}

M. A. Zuluaga, M. Hernandez Hoyos, Eduardo Enrique Davila Serrano, L. F. Uriza, M. Orkisz

\section{- To cite this version:}

M. A. Zuluaga, M. Hernandez Hoyos, Eduardo Enrique Davila Serrano, L. F. Uriza, M. Orkisz. A fast lesion registration to assist coronary heart disease diagnosis in CTA images. International Conference on Computer Vision and Graphics, Sep 2012, Warsaw, Poland. pp.710-717, 10.1007/978-3-642-335648_85. hal-00829657

\section{HAL Id: hal-00829657 https://hal.science/hal-00829657}

Submitted on 30 May 2018

HAL is a multi-disciplinary open access archive for the deposit and dissemination of scientific research documents, whether they are published or not. The documents may come from teaching and research institutions in France or abroad, or from public or private research centers.
L'archive ouverte pluridisciplinaire HAL, est destinée au dépôt et à la diffusion de documents scientifiques de niveau recherche, publiés ou non, émanant des établissements d'enseignement et de recherche français ou étrangers, des laboratoires publics ou privés. 


\title{
A FAST LESION REGISTRATION TO ASSIST CORONARY HEART DISEASE DIAGNOSIS IN CTA IMAGES
}

\author{
Maria A. Zuluaga ${ }^{1,2}$, Marcela Hernández Hoyos ${ }^{2}$, Julio C. Dávila ${ }^{3}$, \\ Luis F. Uriza ${ }^{4}$, and Maciej Orkisz ${ }^{1}$ \\ ${ }^{1}$ Université de Lyon, CREATIS; CNRS UMR5220; INSERM U1044; INSA-Lyon; \\ Université Lyon 1, France \\ ${ }^{2}$ Grupo Imagine, GIB, Universidad de los Andes, Bogotá, Colombia \\ ${ }^{3}$ DIME, Clínica Neurocardiovascular, Cali, Colombia \\ ${ }^{4}$ Hospital Universitario San Ignacio; Pontificia Universidad Javeriana, Bogotá, \\ Colombia
}

The final version of the paper is available from:

http://link.springer.com/chapter/10.1007/978-3-642-33564-8_85

\begin{abstract}
This work introduces a $3 \mathrm{D}+\mathrm{t}$ coronary registration strategy to minimize the navigation among cardiac phases during the process of ischaemic heart disease diagnosis. We propose to register image subvolumes containing suspected arterial lesions at two cardiac phases, instead of performing a registration of the complete cardiac volume through the whole cardiac cycle. The method first automatically defines the extent of the sub-volumes to be aligned, then the registration is performed in two steps: a coarse rigid alignment and a deformable registration. Our method provides comparable results and is computationally less expensive than previous approaches that make use of larger spatial and temporal information.
\end{abstract}

\section{Introduction}

Ischaemic heart disease (IHD) is the main cause of mortality worldwide. Although invasive coronary angiography still remains the clinical gold standard in its diagnosis, computed tomography angiography (CTA) has dramatically progressed so that a reliable visualization of the coronary artery lumen and the identification of coronary plaques are now feasible. However, the diagnostic value of the cardiac CTA images depends on the image quality, which can be strongly affected by the coronary artery motion during the heart beat. Motion patterns differ between the left and right coronary systems and often also between proximal and distal portions of the same artery. The velocity of the arteries also varies along the cardiac cycle and among patients. Such heterogeneous movements and deformations of the coronary arteries lead to motion correction problems and motion artifacts in CTA images. Consequently, the clinicians use at least two 
cardiac phases (time points), typically one at the diastole and another one at the systole, in order to find an optimal representation of each arterial segment and create a mental image of the whole coronary tree. Searching for every structure of interest in each evaluated phase is time consuming and prone to errors.

An alternative to reduce the navigation through the cardiac cycle might be an automatic registration of the arteries at different time points. However, this is a challenging task. The correspondence and alignment ambiguities are inherent to the task of automatically registering $3 \mathrm{D}$ images of tubular shapes [1]. The registration process is further complicated by the fact that vessels are typically surrounded by larger organs that swamp the similarity metric. In a first attempt [2], arterial landmarks have been followed through different time points of the cardiac cycle, using local vascular characteristics. The reported results are interesting, but the method has only been tested on simulated data and on a few points in two real sequences. More recently, a non-rigid registration strategy has been adapted to obtain a $4 \mathrm{D}$ deformation model of the coronary arteries on CTA images at different phases [3. A non-rigid image registration algorithm has also been used to segment and track the coronary arteries in $3 \mathrm{D}+\mathrm{t}$ sequences [4]. Both approaches have been aimed at estimating the coronary motion in order to subsequently exploit it in surgery planning. Since such a procedure is carried out "off-line", the computational time has not been a critical factor. In applications such as daily clinical diagnosis faster methods are required.

In this paper, we propose a $3 \mathrm{D}+\mathrm{t}$ coronary artery registration strategy that minimizes the navigation and facilitates the evaluation of coronary lesions. Our approach mimics the procedure followed by physicians: after detecting a potential lesion in a particular time point of the cardiac cycle, the physician seeks the lesion in another time point to confirm the initial diagnosis. For this matter, only specific volumes of interest (VOI) in the arteries containing potential lesions are registered instead of the whole CTA volume. We demonstrate that our proposal can achieve good results without requiring information of the complete cardiac volume nor of the complete cardiac cycle.

\section{The Method}

The method first automatically defines the extent of the sub-volumes to be aligned. The aim of this step is to construct a VOI that encompasses the lesioned vessel segment at two different time points to be registered. Then the registration is performed in two steps. A coarse rigid alignment first reduces the displacement between the structures of interest (vessels with lesions) in both images. Subsequently, a deformable registration is applied to capture vessel deformations. Further details of each stage are provided in the subsequent sections.

\section{$2.1 \quad$ VOI Definition}

Let $I_{M}(x)$ denote the image at one time point, which will be locally deformed to fit the image from another time point, denoted $I_{F}(x)$. The below-described 
procedure is repeated for each structure of interest (i.e. potential lesion) located in $I_{F}$. The identification of the structures of interest is not the focus of this paper and can be performed either manually or by means of a lesion detection algorithm [65].

We define a VOI $I_{F}^{*}$ that is expected to be discriminant, i.e. contain enough information about the lesioned vessel, so that the latter can be unambiguously identified. The algorithm seeks for the volume that best matches $I_{F}^{*}$ within a VOI of $I_{M}$ denoted $I_{M}^{*}$. The size of both $I_{F}^{*}$ and $I_{M}^{*}$ is a trade-off. While a too small $I_{F}^{*}$ generally leads to the so called aperture problem, a too large $I_{F}^{*}$ is likely to contain a predominant proportion of other structures (e.g. heart ventricles) that may swamp the similarity metric. Similarly, $I_{M}^{*}$ is expected to entirely encompass the structure of interest defined in $I_{F}^{*}$, but be not too large to avoid long computations and possible confusion with other similar structures located nearby.

For this purpose, we construct a parallelepipedal $I_{F}^{*}$ by adding up a thickness $D_{F}$ around the bounding box of the suspected lesion. Similarly, the construction of $I_{M}^{*}$ starts by mapping the spatial location of $I_{F}^{*}$ to $I_{M}$, then a thickness $D_{M}$ is added to build up $I_{M}^{*}$. This parameter corresponds to the maximum displacement magnitude observed on a coronary branch during the cardiac motion [7]. Both thickness parameters $D_{F}$ and $D_{M}$ were determined empirically, then fixed for the remaining experiments.

\subsection{VOI Registration}

Let us first recall that image registration seeks to deform one image, denoted the moving image, to fit another image, denoted the fixed image, by applying a transformation $\mathbf{T}(x)$ to the moving image. The registration problem is formulated as an optimization problem that seeks to minimize a metric $\mathcal{S}$ with respect to the transformation $\mathbf{T}(x)$. Under this premise, our registration stage is a combination of two transformations $\mathbf{T}(x)$ : a (coarse) rigid one and a deformable one.

The coarse rigid registration algorithm is applied to perform an initial alignment of $I_{F}^{*}$ and $I_{M}^{*}$. Its result is used as an input to a subsequent stage where deformations of the lesioned vessels are captured. The deformable registration consists of a non-rigid transform using a free form deformation (FFD) model based on B-cubic splines [8]. The rigid registration is performed at a single resolution, whereas the deformable registration uses a coarse-to-fine multi-resolution strategy. For every resolution, at most 500 iterations are executed by an adaptive stochastic gradient descent optimizer [9]. On every optimization iteration, 2048 samples are obtained from $I_{F}^{*}$ using a random sampler 9 ] and a linear interpolator is used to evaluate the transformed $I_{M}^{*}$ for both rigid and deformable registrations. A $3^{\text {rd }}$ order B-spline interpolator is employed to apply the final deformation transform to $I_{M}^{*}$.

The above-described framework can be used with various similarity metrics $\mathcal{S}$ at both rigid and non-rigid registration stages. The choice was done by a careful empiric evaluation, which will be described in the next sections. 


\section{Experimental Setup}

\subsection{CTA Data}

A total of 92 images from patients undergoing CT coronary angiography at the Hôpital Louis Pradel (Bron, France) were used. The datasets were acquired on a 64-row CTA scanner (Brilliance 64 - Philips Healthcare, Cleveland, OH) with a standard scan protocol. According to their quality, images were classified as poor (25), moderate (26) or good (41). This information was used to assess the performance of the method as a function of the image quality. Twenty paired datasets (systole/diastole pairs) were randomly selected and vessel centerlines were manually traced on three arteries: the right coronary artery (RCA), left anterior descending artery (LAD) and left circumflex artery (LCX). The annotated pairs of centerlines were used to evaluate the quality of the registration by measuring the distance between the reference and the registered artery. The remaining datasets were used in additional optimization and evaluation stages of the work.

\subsection{Experiments}

We first tested various values of the parameters $D_{M}$ and $D_{F}$, respectively ranging from 1.0 to $2.5 \mathrm{~cm}$ and from 0.5 to $1.5 \mathrm{~cm}$. $I_{F}^{*}$ and $I_{M}^{*}$ were respectively built in end-diastolic and end-systolic images, after a manual choice of the arterial segments of interest. These were selected from three coronary arteries (RCA, LAD and LCX) in the proximal (P), distal (D) and intermediate (O) part of each artery. The selection of $D_{M}$ and $D_{F}$ was assessed through a visual inspection by means of a user interface that allows the user to simultaneously navigate in both images 10 .

Subsequent experiments aimed at the selection of the most appropriate similarity metric amongst the following: Mean Squared Differences (MSD), Normalized Correlation Coefficient (NCC), Mutual Information (MI) and Normalized Mutual Information (NMI). For this matter, 90 pairs $I_{F}^{*}, I_{M}^{*}$ were generated: 3 locations per artery on 3 arteries in 10 datasets.

Once a metric selected, an experiment was performed to evaluate whether the direct registration of two time points (end-systole and end-diastole) degrades the quality of the results compared to an incremental registration strategy [34, in which a time point $t$ is registered to time point $t+1$, the resulting transform is used to initialize the registration between phases $t$ and $t+2$ and so on. From the available data, two datasets containing all the cardiac time points were used for incremental registration. Afterwards, a direct registration was performed between time points $40 \%$ and $75 \%$ to compare with the results of the incremental registration.

Registration results assessment was done by evaluating the displacement between the registered lesioned vessel and the corresponding vessel in the fixed image. For this matter, we used previously annotated vessel centerlines as an evaluation criterion. The transformation obtained from the registration of $I_{F}^{*}$ 
and $I_{M}^{*}$ was used to deform the centerline from $I_{M}^{*}$. The distance between the deformed centerline from $I_{M}^{*}$ and the one from $I_{F}^{*}$ was then measured as an indicator of vessel displacement. Let $C_{F}$ be the vessel centerline from $I_{F}^{*}$ and $C_{M}$ the deformed centerline from $I_{M}^{*}$, the average distance between centerlines [4] is defined as:

$$
\begin{aligned}
D\left(I_{F}^{*}, I_{M}^{*}, \mathbf{T}\right)= & \frac{1}{N_{F}} \sum_{i=1}^{N_{F}}\left\|\mathbf{v}_{i}-l\left(\mathbf{v}_{i}, \mathbf{T}\left(I_{M}^{*}\right)\right)\right\| \\
& +\frac{1}{N_{M}} \sum_{j=1}^{N_{M}}\left\|\mathbf{p}_{j}-l\left(\mathbf{p}_{j}, I_{F}^{*}\right)\right\|,
\end{aligned}
$$

where $N_{F}$ and $N_{M}$ are the total numbers of vertices in $C_{F}$ and $C_{M}$, respectively. For each vertex $\mathbf{v} \in C_{F}$, the function $l\left(\mathbf{v}, \mathbf{T}\left(I_{M}^{*}\right)\right)$ calculates the closest vertex to $\mathbf{v}$ on $C_{M}$. Similarly, for each vertex $\mathbf{p} \in C_{M}$, the function $l\left(\mathbf{p},\left(I_{F}^{*}\right)\right)$ calculates the closest vertex to $\mathbf{p}$ on $C_{F}$.

\section{Results and Discussion}

VOI selection. Results obtained from a visual inspection of the generated VOIs $I_{M}^{*}$ showed that $D_{M}=1$ and $1.5 \mathrm{~cm}$ were not enough to capture the movement between end-diastole and end-systole. VOIs formed with $D_{M}=2$ and $2.5 \mathrm{~cm}$ succeeded in always containing the structure of interest. Since our goal was to reduce the amount of processed information $D_{M}=2 \mathrm{~cm}$ was selected. Visual inspection of different $I_{F}^{*}$ VOIs showed that $D_{F} \geq 1 \mathrm{~cm}$ achieves the construction of a VOI with sufficient information. In this case, we preferred to conservatively keep a value larger than the lower limit and defined $D_{F}=1.5 \mathrm{~cm}$ for the remaining experiments.

Time point registration. Registration results in terms of average centerline distance (Eq. 1) using different metrics are presented in Figure 1. As a reference, the initial displacement of the arteries (no registration) was also computed. In general, the best results were obtained when using mutual information, so this metric was kept for the subsequent experimentations.

An analysis of the final vessel displacement in terms of image quality (Fig. 2) showed that the initial displacement has a higher incidence in the results than the image quality. The algorithm performs well on poor quality images but cannot recover excessively large displacements. We consider that the registration does not compensate the initial displacement when the final distance between centerlines is above the average maximum vessel diameter.

Table 1 compares the results obtained by direct and incremental frame registration on two datasets. In 9 cases the direct frame registration outperforms incremental frame registration, while the latter is better in the remaining 9 . Although the incremental approach performs better in most of the cases where the initial displacement between vessels is large (as in RCA and LCX segments), the method does not perform well for very large distances, where the direct approach also fails. From the obtained results it is not possible to say that one of the methods is better than the other. The incremental registration tends to produce smoother images (Figure 3) than the direct approach and to introduce distortion of important structures such as calcified plaques. This can be explained by 


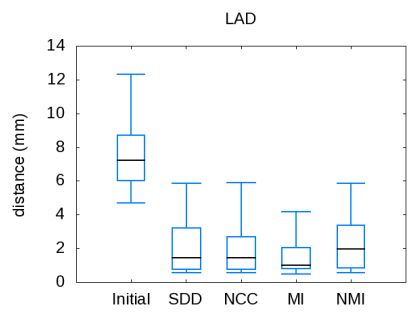

(a)

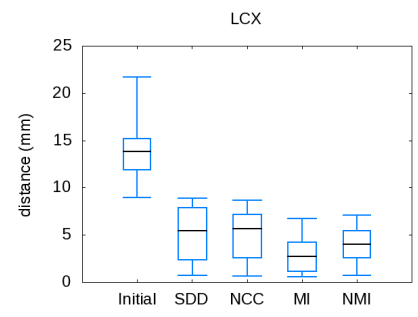

(b)

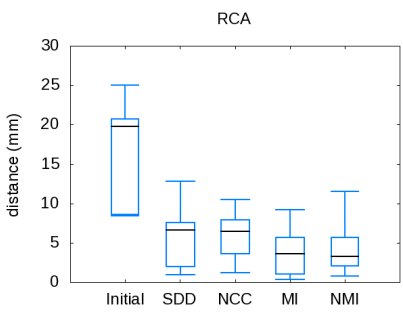

(c)

Fig. 1. Metric evaluation. Initial and final vessel centerline average distance $(\mathrm{mm})$ after non-rigid registration using SSD, NCC, MI and NMI metrics for (a) LAD, (b) LCX and (c) RCA arteries.

Table 1. Direct vs. incremental registration. Distance $(\mathrm{mm})$ between the centerline at the end-diastole and the end-systole before applying any transformation (BT) and after directly (DR) and incrementally (IR) registering three segments of the RCA, LAD and LCX.

\begin{tabular}{c|ccc|ccc}
\hline \multirow{2}{*}{ Segment } & \multicolumn{3}{|c}{ Dataset I } & \multicolumn{3}{c}{ Dataset II } \\
\cline { 2 - 7 } & BT & DR & IR & BT & DR & IR \\
\hline \hline RCA P & 9.18 & 1.20 & 3.61 & 14.98 & 0.55 & 0.50 \\
RCA D & 5.42 & 1.78 & 1.26 & 18.25 & 5.01 & 4.60 \\
RCA O & 6.31 & 1.31 & 2.48 & 15.03 & 7.82 & 6.68 \\
LAD P & 9.08 & 0.44 & 0.55 & 10.90 & 0.49 & 0.75 \\
LAD D & 7.98 & 1.96 & 0.98 & 8.67 & 0.52 & 0.68 \\
LAD O & 8.03 & 0.68 & 1.00 & 10.52 & 0.49 & 0.83 \\
LCX P & 13.33 & 0.73 & 1.15 & 14.21 & 1.03 & 0.80 \\
LCX D & 13.27 & 2.16 & 0.87 & 14.93 & 3.30 & 2.92 \\
LCX O & 13.31 & 1.54 & 1.07 & 14.72 & 2.81 & 2.75 \\
\hline
\end{tabular}



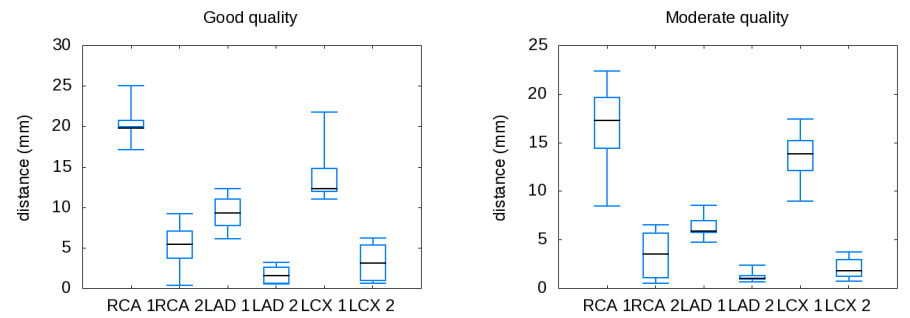

(a)

(b)

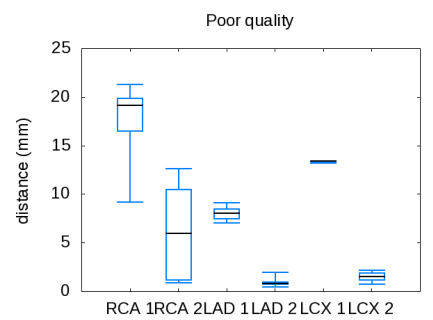

(c)

Fig. 2. Effect of image quality in the registration results - Final vessel centerline average distance $(\mathrm{mm})$ in good (a) moderate (b) and poor (c) quality images. On each plot, 1 denotes initial displacement of the artery, and 2 the final one. 
the higher number of deformations applied in the incremental registration approach. The overall process time for an artery segment was of 6 min in the direct approach and of 37 min for the incremental approach using a Pentium 4 with $3 \mathrm{GHz}$ and $4 \mathrm{~GB}$ RAM. We can state that the direct approach is comparable to the incremental one, in terms of the final vessel displacement results, while remaining computationally less expensive.

\section{Conclusions}

We have presented an approach for the registration of potential vascular lesions in order to speed up the diagnosis process of IHD. The proposed approach registers the potential lesion and its surroundings to a corresponding vessel segment of the image reconstructed at a different time point. The quality of the results and the computational times obtained on a standard desktop PC make us believe that it is feasible to apply this approach in clinical practice.

Future work will focus in defining a metric to more formally validate the values of $D_{F}$ and $D_{M}$, as well as in combining the method with a lesion detection algorithm [6]5] to provide the complete diagnosis pipeline.

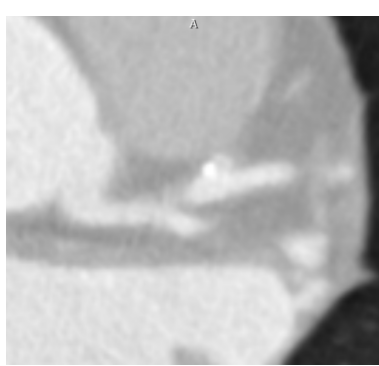

(a)

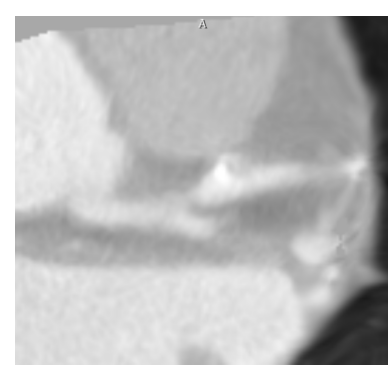

(b)

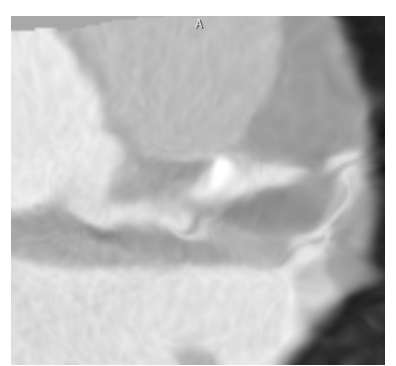

(c)

Fig. 3. Direct vs. incremental registration. The calcification and the vessel shape in (a) are better conserved with direct registration (b). Incremental registration results (c) are more blurred.

\section{Acknowledgements}

This work has been partly funded by ECOS Nord C11S01, Uniandes Interfacultades 06-2010 and Colciencias 1204-519-28996 grants.

\section{References}

1. Aylward, S.R., Jomier, J., Weeks, S. and Bullit, E.: Registration and analysis of vascular images. Int. J. Comput. Vision, 55(2), 123-138 (2003) 
2. Laguitton, S., Boldak, C., Bousse, A., Yang, C. and Toumoulin, C.: Temporal tracking of coronaries in MSCTA by means of 3D geometrical moments. In 28th IEEE EMBS Conf., New York City (USA) 924-927 (2006)

3. Metz, C.T., Schaap, M., Klein, S., Neefjes, L.A., Capuano, E., Schultz, C., van Geuns, R.J., Serruys, P.W., van Walsum, T. and Niessen, W.J.: Patient specific 4D coronary models from ECG-gated CTA data for intra-operative dynamic alignment of CTA with X-ray images. In 12th MICCAI, London (UK), LNCS 5761, 369-376 (2009)

4. Zhang, D.P., Edwards, E., Mei, L., and Rueckert, D.: 4D motion modeling of the coronary arteries from CT images for robotic assisted minimally invasive surgery. In SPIE Med. Imaging, Orlando (USA), vol. 7259 (2009)

5. Zuluaga, M.A., Hush, D., Delgado Leyton, E.J.F., Hernández Hoyos, M. and Orkisz, M.: Learning from only positive and unlabeled data to detect lesions in vascular CT images. In MICCAI, LNCS 6893 Part III, 9Ü16 (2011)

6. Zuluaga, M.A., Magnin, I.E., Hernández Hoyos, M., Delgado Leyton, E.J.F., Lozano, F., and Orkisz, M.: Automatic detection of abnormal vascular cross-sections based on Density Level Detection and Support Vector Machines, Int. J. Computer Assist. Radiol. Surg., 6(2), 163-174 (2011)

7. Shechter, G., Resar, J.R. and McVeigh, E.R.: Displacement and velocity of the coronary arteries: cardiac and respiratory motion. IEEE Trans. Med. Imaging, 23(3), 369Ü375 (2006)

8. Rueckert, D., Sonoda, L.I., Hayes, C., Hill, D.L.G., Leach, M.O. and Hawkes, D.J.: Non-rigid registration using free-form deformations: application to breast MR images. IEEE Trans. Med. Imaging. 18(8), 712 Ü721 (1999)

9. Klein, S., Staring, M., Murphy, K., Viergever, M.A. and Pluim, J.P.W.: elastix: a toolbox for intensity-based medical image registration. IEEE Trans. Med. Imaging, 29(1), $196 \tilde{\mathrm{U}} 205$ (2010)

10. Mattes, D., Haynor, D.R., Vesselle, H., Lewellen, T.K. and Eubank, W.: PET-CT image registration in the chest using free-form deformations. IEEE Trans. Med. Imaging, 22(1), 120 Ü128 (2003) 